\title{
Treatment Pattern and Outcomes in Newly Diagnosed Multiple Myeloma Patients Who Did Not Receive Autologous Stem Cell Transplantation: A Real-World Observational Study
}

\section{Treatment pattern and outcomes in patients with multiple myeloma}

\author{
Jianming He (D) - Luke Schmerold • Rian Van Rampelbergh • Lugui Qiu • Ravi Potluri • \\ Anandaroop Dasgupta $\cdot$ Lin Li · Yunan Li · Peter Hu $\cdot$ Sepideh Nemat $\cdot$ Steven S. Smugar • \\ Paul Zeltzer · Carlos Appiani · Qing Li · Maneesha Mehra · Ute Richarz \\ Received: August 14, 2020 / Accepted: October 21, 2020 / Published online: November 19, 2020 \\ (C) The Author(s) 2020
}

\section{ABSTRACT}

Introduction: The objective of this study was to describe the treatment patterns among patients with newly diagnosed multiple myeloma (MM) who had not received autologous stem cell transplantation (ASCT). It further compares the safety and clinical outcomes across different frontline regimens as well as explores whether treatment duration predicts outcomes.

Methods: Patients with MM ( $>45$ years) who had not received ASCT were retrospectively

Electronic Supplementary Material The online version of this article (https://doi.org/10.1007/s12325020-01546-0) contains supplementary material, which is available to authorized users.

J. He · M. Mehra

Janssen Global Services LLC, Raritan, NJ, USA

L. Schmerold · R. Potluri · A. Dasgupta

SmartAnalyst Inc., New York, NY, USA

R. Van Rampelbergh

Janssen R\&D, Beerse, Belgium

L. Qiu

Institute of Hematology and Blood Diseases

Hospital, Chinese Academy of Medical Sciences and

Peking Union Medical College, Tianjin, China

L. Li · Y. Li

Xian Janssen Pharmaceutical Ltd, Beijing, China identified from the US SEER-Medicare (Jan 2007-Dec 2016) and Optum (Jan 2007-Sep 2018) databases. Cox proportional hazard models were used to compare overall survival (OS) among bortezomib + lenalidomide + dexamethasone regimen (VRd), lenalidomide + dexamethasone regimen (Rd), cyclophosphamide + bortezomib + dexamethasone regimen (CyBorD), bortezomib + dexamethasone regimen $(\mathrm{Vd})$, and other bortezomib-containing therapies based on propensity score matching. To address immortal time bias, timefixed and time-dependent Cox models were employed to estimate the association of longer frontline treatment exposure with outcomes.

P. $\mathrm{Hu}$

Janssen R\&D, Raritan, NJ, USA

S. Nemat

Janssen R\&D, High Wycombe, UK

S. S. Smugar · P. Zeltzer · C. Appiani · Q. Li

Janssen R\&D, Titusville, NJ, USA

U. Richarz ( $\square)$

Janssen Global Medical Affairs, Cilag, Zug,

Switzerland

e-mail: urichar1@its.jnj.com 
Results: Mean (standard deviation; SD) age was 71 (9.8) years; and $49.51 \%$ were women. Bortezomib and lenalidomide-based combinations were the most common treatment modalities. After matching, the HR $(95 \% \mathrm{CI})$ of OS by frontline therapies comparing VRd with Vd was $0.76(0.66,0.86)$, CyBorD was $0.87(0.75,1.05)$, for other bortezomib-based therapies was 0.56 $(0.49,0.64)$, Rd was $0.83(0.73,0.95)$, and for other therapies was $0.70(0.61,0.80)$. Longer frontline treatment duration was associated with better OS for overall frontline [HR $(95 \% \mathrm{CI})$ 0.86 (0.82, 0.90)]; Vd [0.81 (0.74, 0.89)]; CyBorD [0.79 (0.64, 0.98)] and Rd [0.86 (0.78, 0.95)].

Conclusion: Results demonstrated that the frontline therapies prescribed to most patients who did not receive ASCT for MM in the United States were consistent with the NCCN guideline recommendations. Longer frontline treatment duration was associated with improved OS.

Keywords: Newly diagnosed multiple myeloma; Outcomes; Transplant ineligible

\section{Key Summary Points}

Why carry out this study?

Multiple myeloma (MM), a rare

hematologic malignancy, led to 98,437 deaths and 2.1 million disability-adjusted life-years globally in 2016. Healthcare costs for newly diagnosed MM patients has risen over threefold from 2000 to 2014 in the United States.

Although the American Society of Clinical Oncology guidelines recommend initiation of therapy with proteasome inhibitors, immunomodulators, and steroids for patients who are not eligible for autologous stem cell transplantation (ASCT), outcomes with these therapies are dependent on various factors. In addition, real-world studies evaluating the effects of these treatments are scarce.
The present study evaluated the treatment patterns and outcomes among newly diagnosed MM patients who had not received ASCT, from the Surveillance, Epidemiology, and End Results (SEER)Medicare and Optum databases. The study further compared the safety and clinical outcomes among different frontline regimens.

\section{What was learned from the study?}

The median OS of patients receiving Vd, VRd, CyBorD, and Rd therapy was 56.3 months, 112.6 months, 92.9 months, and 79.1 months, respectively. Patients treated with Rd therapy had the longest TTNT (24.3 months).

The study demonstrated that the frontline therapies prescribed to the patients who did not receive ASCT for MM in the United States were consistent with NCCN guideline recommendations. Patients who had received VRd showed better outcomes compared with Rd, Vd, and CyBorD therapies.

\section{DIGITAL FEATURES}

This article is published with digital features, including a summary slide, to facilitate understanding of the article. To view digital features for this article go to https://doi.org/10.6084/ m9.figshare.13096448.

\section{INTRODUCTION}

Accounting for approximately $1 \%$ of all cancers and $15-20 \%$ of hematologic malignancies worldwide, multiple myeloma (MM) is associated with substantial clinical, health-related quality of life and economic burdens that increase as the disease progresses [1, 2]. In 2016, 138,509 new cases of MM were diagnosed, and 98,437 deaths occurred, globally. In the same 
year, MM caused 2.1 million disability-adjusted life-years [3]. The American Cancer Society has estimated 32,270 new MM cases and 12,830 deaths due to MM to be reported in the United States by the end of 2020 [4]. The all-cause healthcare costs for newly diagnosed MM patients have significantly increased, from US\$3263 per patient per month (PPPM) in 2000 to $\$ 14,656$ PPPM in 2014 in the United States [5].

For newly diagnosed patients with MM, eligibility for autologous stem cell transplantation (ASCT) is primarily determined based on the age and comorbidities followed by performance status, frailty, disability, and availability of social and family support. Although median survival has improved for ASCT-ineligible patients in recent years, these patients have a lower survival rate than ASCT-eligible patients. The American Society of Clinical Oncology guidelines recommend initiation of therapy with proteasome inhibitors (PI), immunomodulators (IMiD), and steroids for patients who are ineligible for ASCT [6]. Outcomes with these anti-MM therapies are highly dependent on disease-specific (stage of MM and cytogenetic abnormalities) and patient-specific factors (age, comorbidities, performance status, frailty status, and patient preference) [6]. Combination therapies including category 1 regimen [bortezomib/lenalidomide/dexamethasone (VRd), daratumumab/lenalidomide/dexamethasone (DRd), lenalidomide/low-dose dexamethasone (Rd)] and bortezomib/cyclophosphamide/dexamethasone (CyBorD) are recommended as preferred regimens for newly diagnosed patients who are not ASCT candidates by the National Comprehensive Cancer Network (NCCN) guidelines, which have demonstrated significant improvement on clinical outcomes of MM, including overall survival (OS) and progressionfree survival (PFS) [7-9]. Other recommended options include daratumumab/bortezomib/ melphalan/prednisone (DVMP), carfilzomib or ixazomib combined with Rd, bortezomib/dexamethasone (Vd) and cyclophosphamide/lenalidomide/dexamethasone, and carfilzomib/ cyclophosphamide/dexamethasone [10]. However, real-world studies evaluating the effectiveness of these treatments are scarce.
Therefore, this study was conducted to describe the treatment patterns and outcomes among patients with newly diagnosed MM who had not received ASCT, analyzing data from the Surveillance, Epidemiology, and End Results (SEER)-Medicare and the Optum databases. The study further compared the safety and clinical outcomes across different frontline regimens as well as explored whether treatment duration predicts outcomes.

\section{METHODS}

\section{Data Source}

Data for the present study were obtained from the US population-based SEER registry and Optum [Clinformatics and electronic health records (EHR)] databases. The SEER-Medicare database predominantly contains data of elderly patients ( $\geq 65$ years of age) because of the eligibility criteria of Medicare. To include younger patients who had not received ASCT, additional data were obtained from the Optum databases. Patients aged $\geq 65$ years old in Optum databases were excluded to prevent potential duplication with SEER-Medicare.

The SEER-Medicare database contains clinical, demographic and cause of death information for patients with cancer from SEER, comprised of population-based cancer registries covering approximately $34.6 \%$ of the population of the United States, and related Medicare claims for covered health care services from the time of a person's Medicare eligibility until death. The SEER-Medicare-linked database is usually updated biennially. As of June 2020, the data include all Medicare-eligible persons appearing in the SEER data who were diagnosed with cancer through 2015, and their Medicare claims through 2016.

Optum Clinformatics is an insurance claims database that includes both medical and prescription coverage and includes approximately 15 million covered affiliate lives annually, with over 180 million claims in total. The Optum EHR database includes data for over 80 million patients from over 195 hospitals, and captures 
clinical, operational, and financial information recorded by physicians at the time of care.

\section{Study Design}

This retrospective cohort study included data from 1 January 2007 through 30 September 2018 (SEER-Medicare: 1 January 2007 to December 2016, Optum databases: 1 January 2007 to 30 September 2018). The use of SEER registry and Optum (Clinformatics and EHR) databases was reviewed by the Institutional Review Board (IRB). The study protocol developed for obtaining data from the SEER Medicare database was reviewed and exempted by Advarra IRB (Columbia, MD, USA), as this research project did not involve human patient research.

Patients of either sex above 45 years of age, who were diagnosed with MM [International Classification of Diseases (ICD)-9 203.0, ICD-10 C90.0 and ICD-O-3 9732] and received at least one line of treatment (LOT) with minimum look-back period of 6 months were included. A LOT was identified based on an initial administration of $\geq 1$ agent that continued until $\geq 1$ agent was discontinued for $\geq 60$ days or until a new agent was administered. A regimen containing a PI (e.g., bortezomib) and an IMiD (e.g., lenalidomide) was considered to continue until both the PI and the IMiD were discontinued. Patients without insurance coverage at the time of diagnosis, those being treated with ASCT, or patients with previous history of cancer (including in situ cancers and skin cancers) were excluded. Patients who were diagnosed with another cancer before their first LOT were also excluded, as treatments must address secondary malignancy.

First line of treatment (LOT1) included Rd, VRd, CyBorD, Vd, other bortezomib-containing regimens [e.g., bortezomib + melphalan + prednisolone (VMP) and bortezomib + thalidomide \pm dexamethasone

(VTd)], and other regimens [regimen does not include PI or IMiD, such as melphalan and prednisone (MP)]. In the study period, daratumumab, ixazomib, and carfilzomib were rare. Second line of treatment (LOT2) regimens included lenalidomide mono, lenalidomide combination regimens, bortezomib mono, bortezomib combination regimens, and others (e.g., daratumumab mono and daratumumabbased regimens, ixazomib and ixazomib-based regimens).

\section{Study Assessments}

Index date was defined as the first day of receiving LOT1 treatment. Patient demographic and clinical characteristics were assessed at the start of LOT1. Patient comorbidities at baseline (index date) and during LOT1 were assessed. Incidence rate of comorbidity was reported for LOT1 by the most common comorbidities, i.e., anemia, thrombocytopenia, neutropenia, peripheral neuropathy, diarrhea, nausea, fatigue, secondary cancer (i.e., hematological malignancy, solid tumor), and infusion-related reactions. For peripheral neuropathy, we also reported the incidence rate stratified by patients with and without a history of peripheral neuropathy. Results are presented by frontline treatment and by LOT. Time from index date to death or loss of follow-up/censor, whichever occurred first, was defined as OS and estimated. Time to next treatment (TTNT) was defined as the time from the index date to the start of LOT2; patients were censored at loss to followup or death. The treatment-free interval (TFI) was defined as the duration between the discontinuation of LOT1 and initiation of LOT2/censor/death.

A limitation of the Optum cohort is the incompleteness of the death data, such that a patient's death will not always be captured. To account for this, we conducted a sensitivity analysis for OS in which death was imputed by identifying patients who had a missing death date and had more than 360 days' gap between enrollment end date (Optum claims) or last claim date (Optum EHR) and the study end date. The enrollment end date refers to the last date a patient had continuous insurance enrollment. These patients were assumed to have died on their enrollment end date or last claim date. 


\section{Statistical Analysis}

Descriptive statistics were used to report patient characteristics, treatment duration, TFI, and morbidities reported during the study. The Kaplan-Meier method was used to present OS and TTNT. Propensity score matching (Greedy nearest-neighbor matching with caliper as 0.20 ) models were considered as the base case to compare VRd versus other frontline regimens with age, gender, Charlson comorbidity index (CCI, whether patients have one comorbidity vs. none), presence of heart diseases, and renal disorders at treatment initiation matched. Selection of potential confounders was based on expert opinions made on available baseline patient characteristic variables. Standard mean differences and variance ratios were calculated to determine if propensity score matching was satisfactory (please see Electronic supplementary material). Stratified Cox proportional hazards model on OS and TTNT was used to compare major LOT1 treatments based on matched pairs. Multivariable Cox models were conducted adjusted by age, gender, CCI, presence of heart diseases, and renal disorders at treatment start as sensitivity analysis.

To understand the predictive value of longer treatment duration, median duration of treatment (mDOT) of the LOT1 regimens were calculated, and the hazard ratio (HR) of OS and TTNT between patients in the longer than median treatment duration group (LDG) versus patients in the shorter than median treatment duration group (SDG) were compared based on the Cox proportional hazard models [11, 12], adjusted by age, gender, CCI, presence of heart diseases, and CCI renal disorders. The operational definition of the LDG posits that it will not experience the outcomes (i.e., remain immortal) until mDOT is reached in the respective regimen cohorts. Immortal time bias, if not accounted for, can confer a spurious advantage in outcomes to LDG over SDG. In order to adjust for immortal time bias, we developed three variants of Cox models. Base case analysis controlling for the presence of immortal time was conducted using a time-dependent Cox model. In this model, LDG use during immortal time was classified as SDG. A time-fixed Cox model (sensitivity analysis 1) including immortal time was conducted to estimate hazard ratio. We also used a time-fixed model in which immortal time was excluded from LDG and not added to SDG (sensitivity analysis 2). Those models were consistent with methods proposed by Yang et al. and Suissa $[11,12]$. Furthermore, the doubly robust method was not required for the present analysis as the treatment selection and outcome models have been correctly specified. A $P<0.05$ was considered statistically significant. In order to address multiplicity adjustment in the outcome analyses comparing VRd with other regimens, a Bonferroni-corrected $P$ value threshold of 0.01 was used to identify a statistically significant treatment effect. Our rationale to use Bonferroni correction has been discussed elsewhere [13, 14].

\section{RESULTS}

\section{Baseline Characteristics}

Overall, 17,731 patients were included in the study of which 14,273 patients $(80.49 \%)$ were included from the Optum databases and 3458 patients (19.50\%) from the SEER-Medicare database (Fig. 1). Common regimens administered to the patients in this cohort were $\mathrm{Rd}$ (4358; 24.57\%), Vd (4128; 23.28\%), VRd (2718; $15.32 \%)$, and CyBorD (1225; 6.90\%). The mean (SD) age of the patients was $71.0(9.8)$ years, and patients receiving VRd therapy were the youngest [mean (SD) 68.7 (9.3) years]. Almost half of the patients included in the study were women $(n=8779 ; 49.51 \%)$. The mean (SD) duration between diagnosis of $\mathrm{MM}$ and treatment initiation in all patients was 2.8 (7.3) months. The majority of the patients were insured with Medicare (57.62\%) and nearly a quarter of patients were commercially insured (26.36\%). Hypertension (59.87\%), renal failure $(30.53 \%)$, fluid and electrolyte disorders $(27.33 \%)$, diabetes $(25.37 \%)$, and peripheral neuropathy $(11.16 \%)$ were the most prevalent comorbidities at baseline. Renal failure may be attributed to myeloma. The mean (SD) CCI was 1.9 (2.3) of which the CCI of patients receiving 


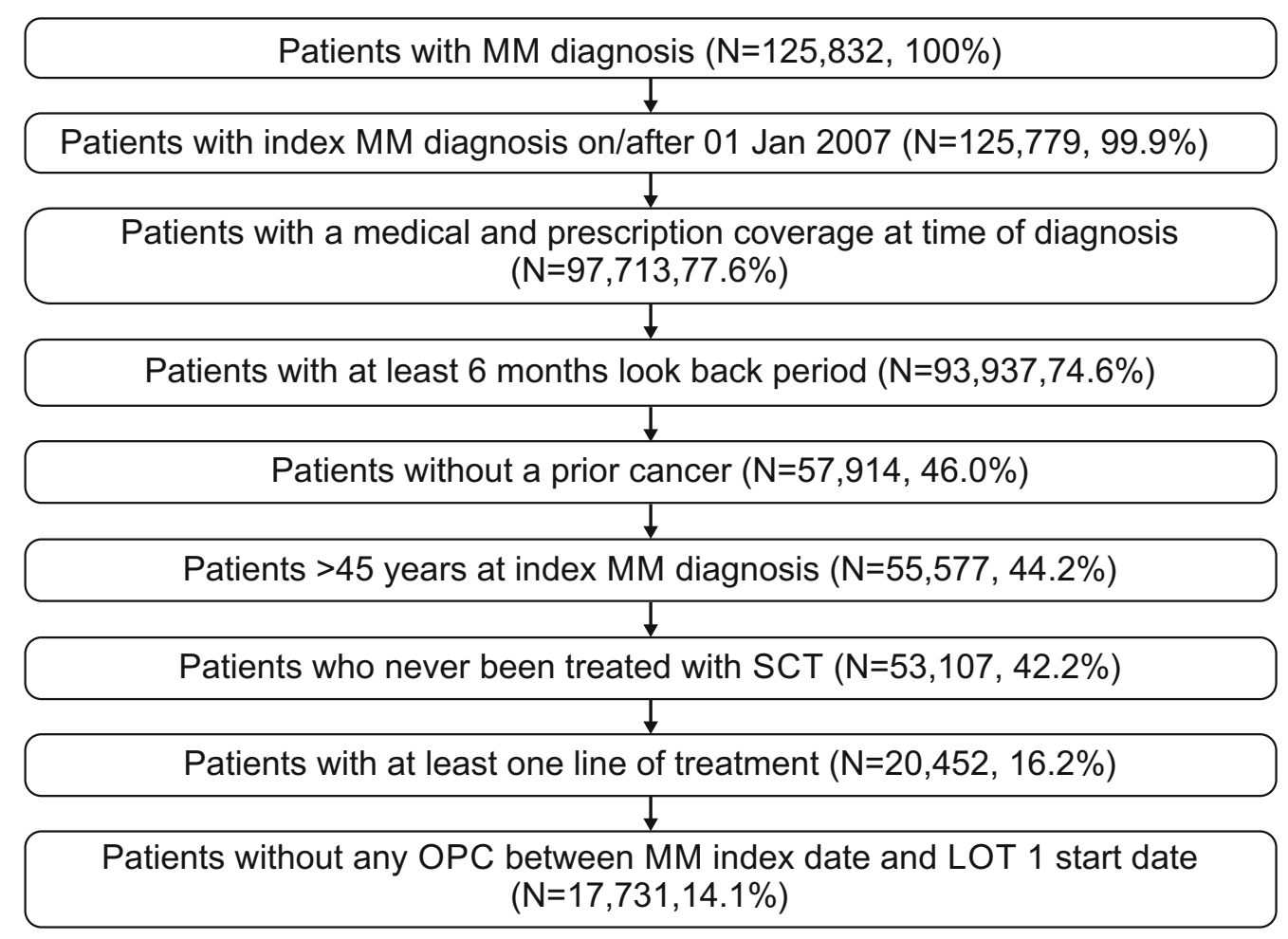

Fig. 1 Patient disposition. $M M$ multiple myeloma, $O P C$ other primary cancer, $L O T$ line of therapy, $S C T$ stem cell transplantation

other regimens (regimen other than PI and IMiD) was the lowest $1.5(2.1)$ and CCI of patients receiving $\mathrm{Vd}$ therapy was the highest 2.6 (2.6) (Table 1), indicating that $\mathrm{Vd}$ is more likely to be used for patients with multiple comorbidities.

\section{Frontline Comorbidities}

Overall, commonly observed comorbidities in LOT1 reported by patients with MM included anemia (52.65\%), nausea (25.18\%), fatigue (21.98\%), peripheral neuropathy (19.63\%), and diarrhea (13.85\%). Among patients with peripheral neuropathy, $37.26 \%$ had a history of being afflicted with peripheral neuropathy while $14.10 \%$ did not (Table 1). The most commonly observed comorbidities among patients treated with bortezomib-containing regimens were anemia $(58.63 \%)$, nausea $(30.30 \%)$ fatigue $(23.26 \%)$, peripheral neuropathy (21.75\%; with history [38.61\%] and without history [16.08\%]), diarrhea (15.94\%), and thrombocytopenia (10.50\%) (Table 1). Among individual regimens, VRd (27.00\%) and Vd $(22.33 \%)$ had the highest rate of peripheral neuropathy. Across the frontline treatment regimens, patients with a history of peripheral neuropathy had a higher risk of experiencing peripheral neuropathy compared to patients without a history of peripheral neuropathy. Although bortezomib-based regimens were associated with elevated peripheral neuropathy, the prevalence was not substantially different than that of $\mathrm{Rd}(16.65 \%)$.

\section{Treatment Duration and Treatment Switch}

Patients treated with VRd therapy had the highest median duration of treatment (181 days), followed by Vd (129 days), and Rd 


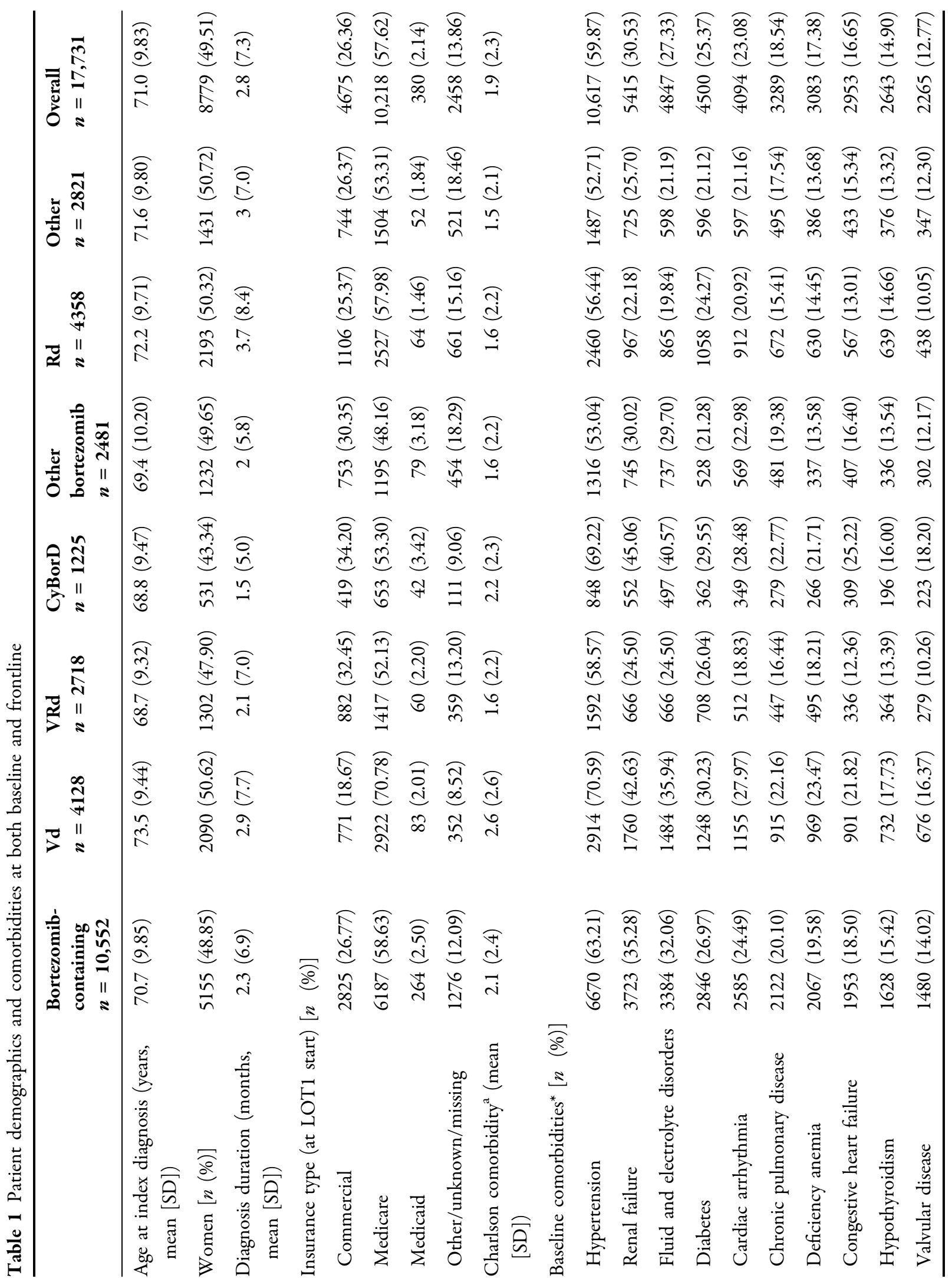




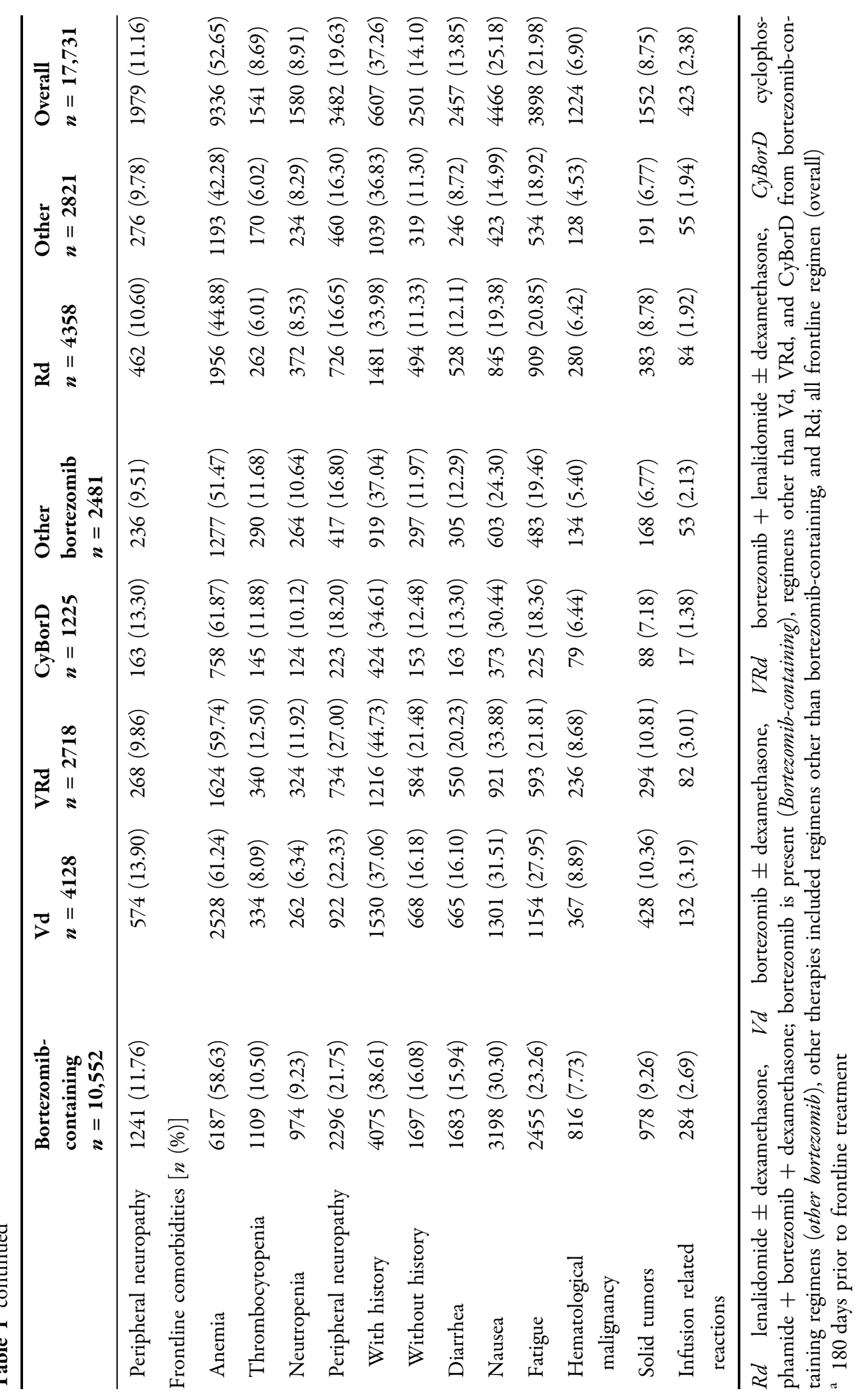




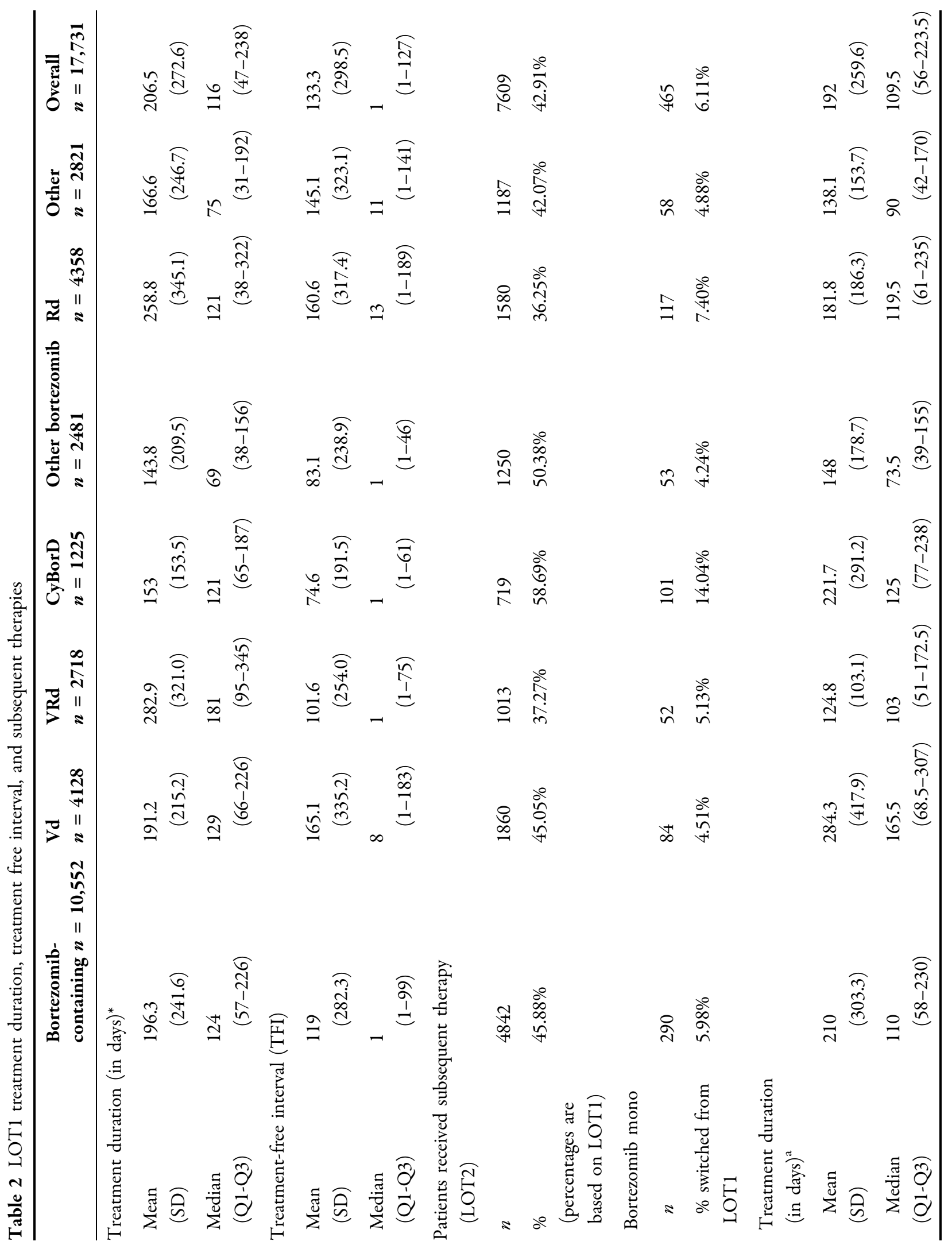




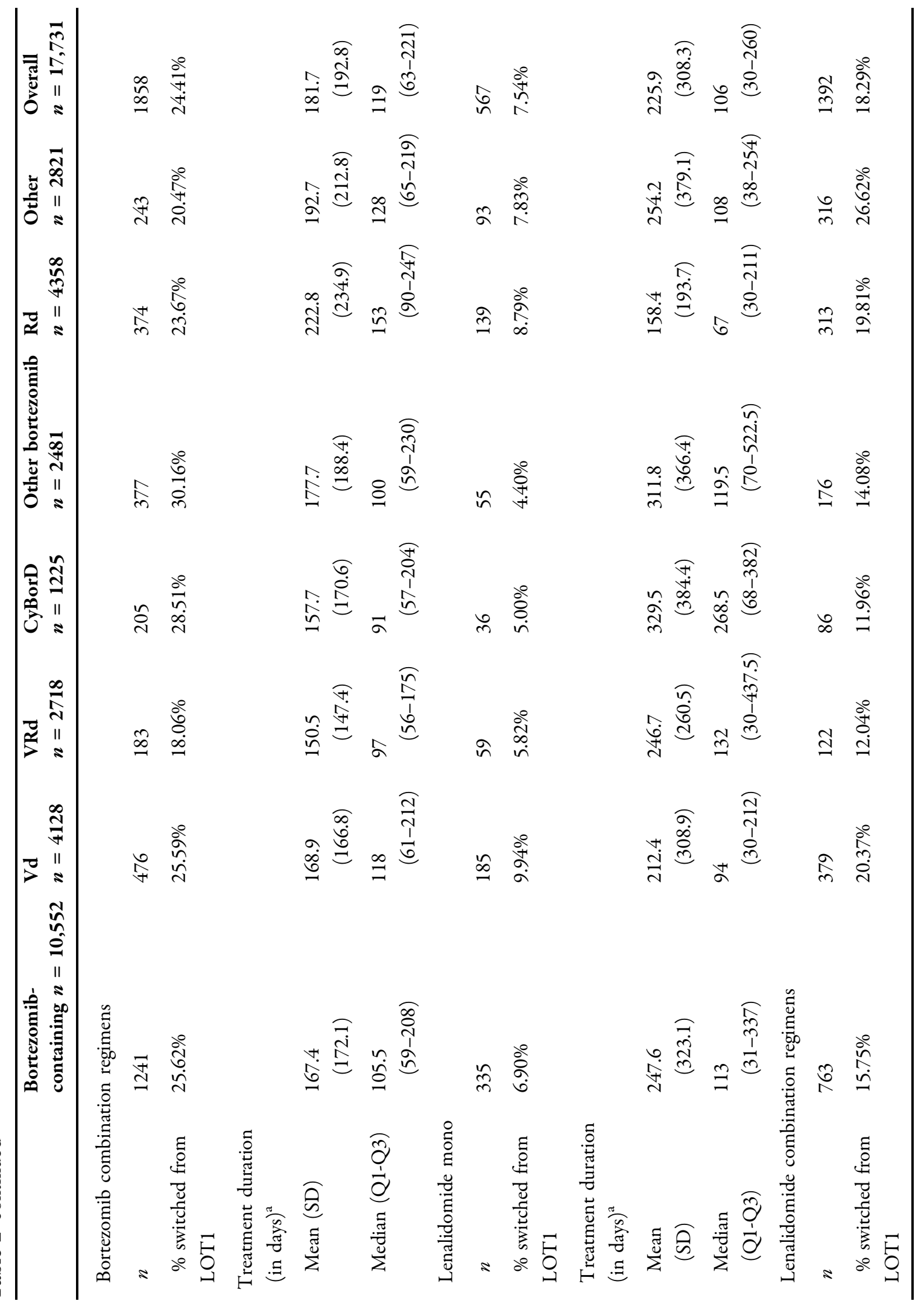




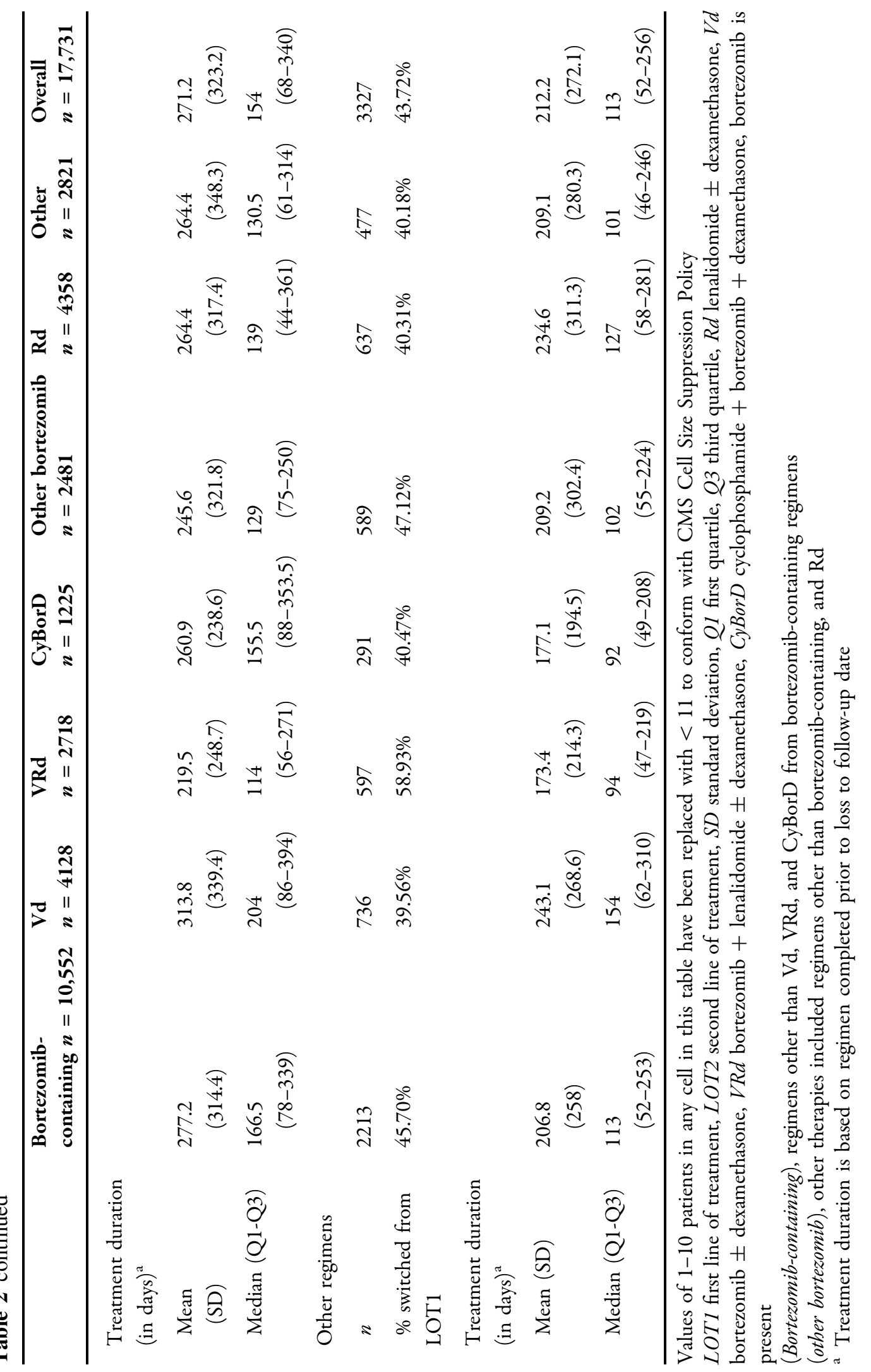


and CyBorD (121 days each) therapies. Only $42.91 \%$ of LOT1 patients moved on to LOT2 (Table 2). Majority of the patients treated with CyBorD therapy $(58.69 \%)$ received subsequent treatment (LOT2). Only $36.25 \%$ of Rd patients received LOT2 (Table 2). The most common overall LOT2 therapies were bortezomib combination regimens $(24.41 \%)$ and lenalidomide combination regimens $(18.29 \%)$. While usage of bortezomib mono (6.11\%) and lenalidomide mono $(7.54 \%)$ was low, the utilization of newer agents such as daratumumab was under $1 \%$ at this period. Among patients who received $\mathrm{Rd}$ as LOT1, $8.79 \%$ continued it in LOT2 while $19.81 \%$ switched to lenalidomide combination regimens. Similarly, among patients who received $\mathrm{Vd}$ as LOT1, $4.51 \%$ continued it in LOT2 while $25.59 \%$ switched to bortezomib combination regimens.

\section{Overall Survival}

The median OS (95\% CI) of patients receiving Vd, VRd, CyBorD, other bortezomib-containing regimens and Rd therapy was 56.3 months (95\% CI 52.0, 60.5), 112.6 months [93.0, not reached (NR)], 92.9 months (71.3, NR), 57.2 months $(52.5,64.5)$ and 79.2 months $(74.1,85.7)$, respectively (Fig. 2). Propensity score matching was considered as the base case. Matching was generally satisfactory with standardized mean difference (SMD) of most variables from most models were less than 0.10 and variance ratios were close to 1 after matching. Only the SMD of age after matching is 0.12 for VRd matched with other treatments. After matching, the HR of OS by frontline therapies comparing VRd versus $\mathrm{Vd}$ was 0.76 (95\% CI 0.66, 0.86), VRd versus CyBorD was 0.87 (95\% CI 0.72, 1.05), VRd versus other bortezomib-based therapies was 0.56 (95\% CI $0.49,0.64)$, VRd versus $\mathrm{Rd}$ was 0.83 $(95 \%$ CI $0.73,0.95)$, and VRd versus other therapies was 0.70 (95\% CI 0.61, 0.80) (Table 3). The results of multivariate analysis were consistent with propensity score matching, with results favoring VRd in both cases. The adjusted HR of OS sensitivity analysis by frontline therapies comparing VRd versus Vd was 0.78 (95\% CI 0.70, 0.86), VRd versus CyBorD was 0.78
(95\% CI $0.68,0.91)$, VRd versus other bortezomib based therapies was 0.58 (95\% CI 0.52 , 0.65 ), VRd versus Rd was 0.74 (95\% CI 0.67 , $0.81)$, VRd versus other therapies was 0.69 (95\% CI $0.62,0.76)$. Sensitivity analysis was conducted with a modified OS to circumvent probable under-reporting of deaths in the Optum databases (Table 3). Results of propensity score matching and multivariate regression for both OS and imputed OS were also generally consistent, reflecting a trend of improved OS by VRd regimen after adjusting for age, gender and baseline comorbidity.

\section{Time to Next Treatment (TTNT) and Treatment-Free Interval (TFI)}

Patients treated with Rd therapy had the longest TTNT (24.3 months; 95\% CI 23.0, 25.8) compared with VRd therapy (20.4 months; 19.1, 22.7), CyBorD therapy (7.0 months; 6.5, 7.6) and Vd therapy (15.4 months; 95\% CI 14.5, 16.5 , Fig. 3). The adjusted HR (95\% CI) of TTNT by frontline therapies comparing VRd versus $\mathrm{Vd}$ was 0.57 (95\% CI 0.50, 0.65), VRd versus CyBorD was 0.28 (95\% CI 0.23, 0.34), VRd versus other bortezomib therapies was 0.27 (95\% CI 0.23, 0.32), VRd versus Rd was 0.97 (95\% CI $0.84,1.11$ ), and VRd versus other therapies was 0.66 (95\% CI 0.61, 0.73) (Table 3). Multivariate analysis results were consistent with propensity score matching. The results generally favored VRd except comparing to Rd.

The longest mean TFI was observed in patients who were treated with Vd therapy (165.1 days), followed by Rd therapy (160.6 days) (Table 2). The TFI was right skewed with half of patients receiving their LOT2 within one month.

\section{Association Between Frontline Treatment Duration and Outcomes}

Patients who received regimens for a longer duration had higher median OS than those who received treatment for a shorter duration [74.5 months vs. 64.5 months, HR (95\% CI) $0.67(0.64,0.70)]$. The median treatment duration of bortezomib-containing regimens and $\mathrm{Rd}$ 


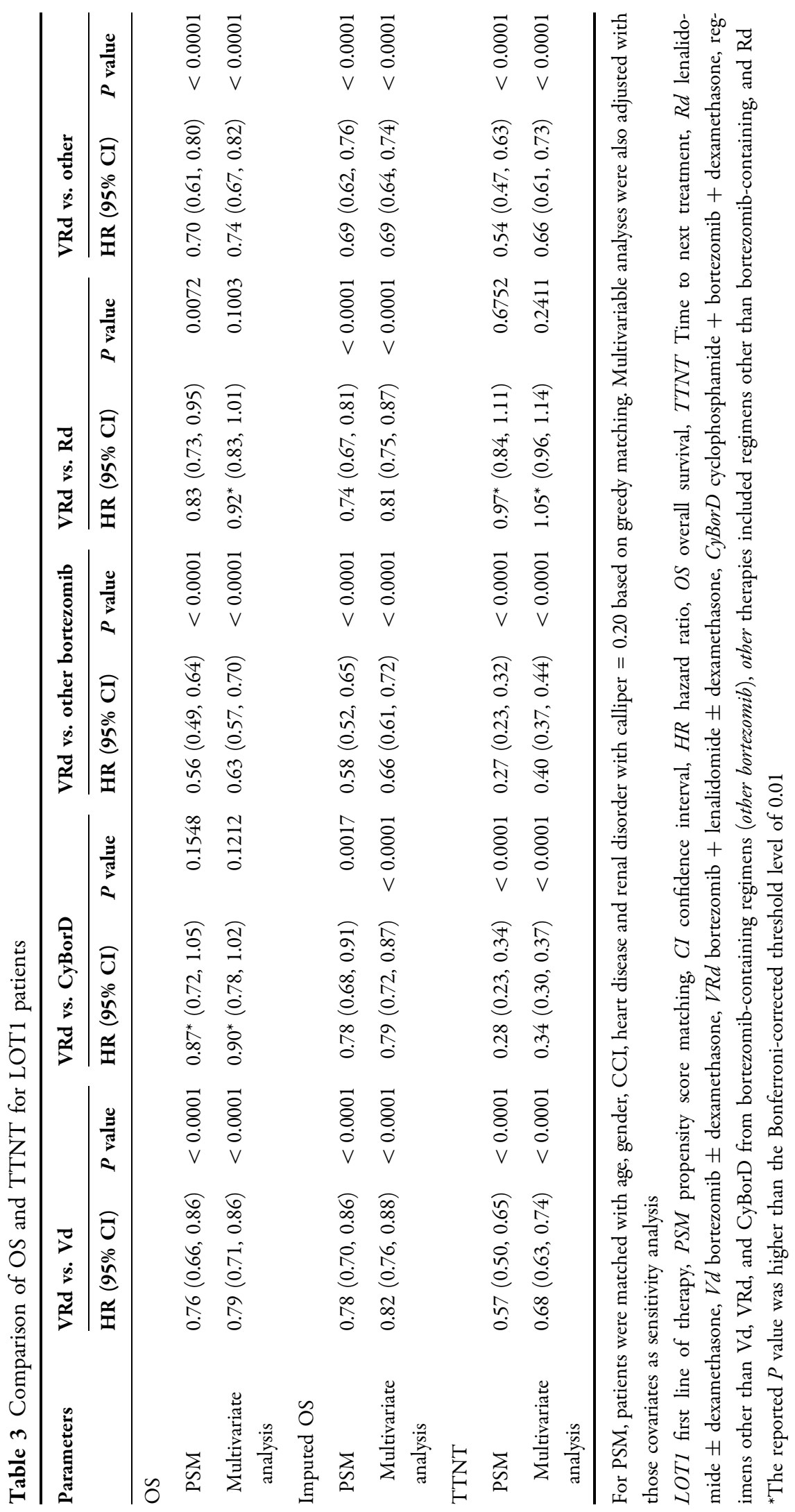




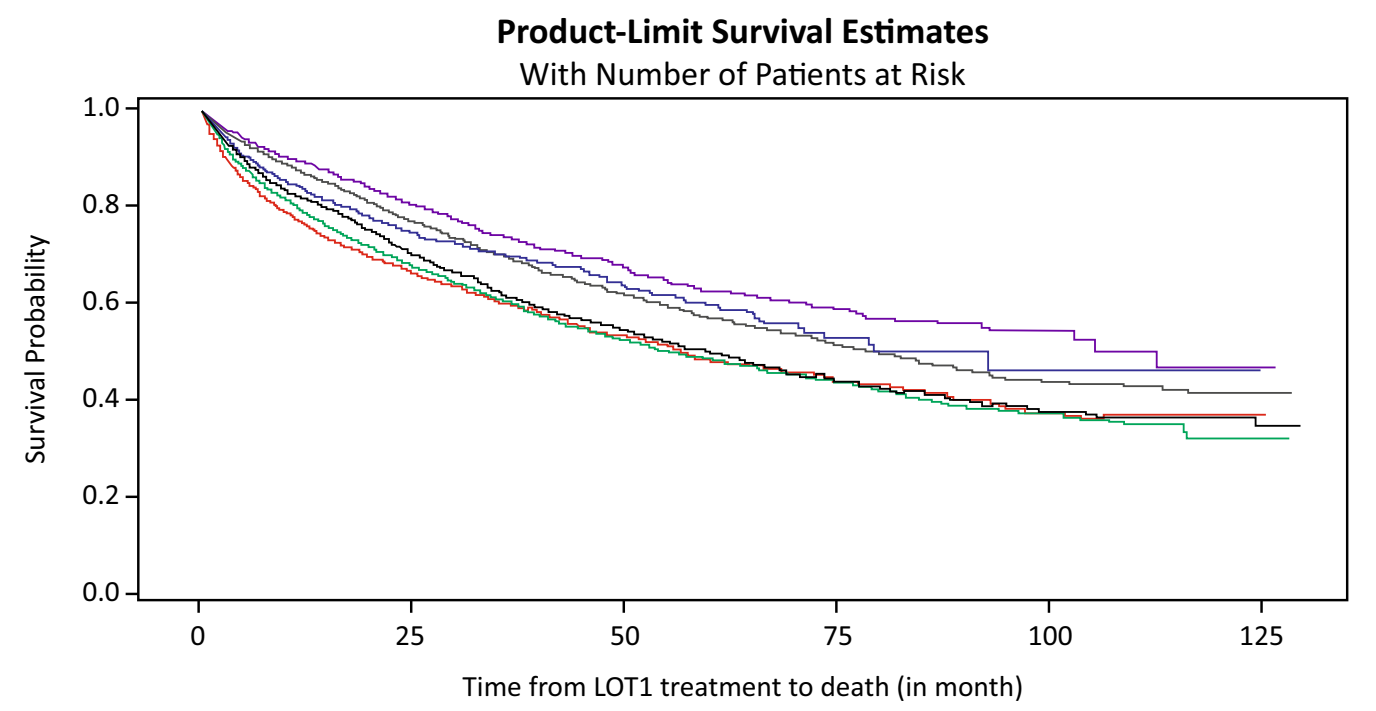

\begin{tabular}{|lll|}
\hline 1. CyBorD & 2. Other bortezomib containing & 3. Other \\
4. Rd & 5. VRd & 6. Vd \\
\hline
\end{tabular}

Patient at risk

$\begin{array}{cccc}1 & 1225 & 663 & 252 \\ 2 & 2481 & 1285 & 686 \\ 3 & 2821 & 1545 & 873 \\ 4 & 4358 & 2636 & 1377 \\ 5 & 2718 & 1405 & 633 \\ 6 & 4128 & 2332 & 1177\end{array}$

Fig. 2 Overall survival. $R d$ lenalidomide \pm dexamethasone, $V d$ bortezomib \pm dexamethasone, $V R d$ bortezomib + lenalidomide \pm dexamethasone, CyBorD cyclophosphamide + bortezomib + dexamethasone; bortezomib

were similar (124 and 121 days, respectively). After accounting for immortal time bias, the time-dependent and time-fixed Cox models showed that the LDG was associated with better OS in general. The time-dependent adjusted Cox model yielded HRs (95\% CI) of OS comparing LDG versus SDG as $0.81(0.74,0.89), 0.87$ $(0.74,1.01), 0.79(0.64,0.98), 1.11(0.98,1.25)$, $0.86(0.78,0.95), 0.86(0.82,0.90)$ for Vd, VRd, CyBorD, other bortezomib-containing regimens, Rd and overall LOT1, respectively. Results from both time-fixed Cox model including immortal time (sensitivity analysis 1) and excluding immortal bias (sensitivity analysis 2) showed significant favorable outcomes for longer treatment associated with Vd, VRd,
CyBorD, Rd and overall LOT1. The association was not significant for other bortezomib-containing regimens and other regimens. However, base case and sensitivity analyses conducted with imputed OS demonstrated more pronounced results; HR (95\% CI) of imputed OS comparing LDG versus SDG based on the timedependent Cox model was $0.62(0.57,0.67)$, $0.57(0.51,0.64), 0.6(0.52,0.71), 0.63(0.57$, $0.70), 0.6(0.56,0.65), 0.58(0.56,0.61)$, for $\mathrm{Vd}$, VRd, CyBorD, other bortezomib-containing regimens, Rd, and overall LOT1 regimens, respectively. Conversely, the HR $(95 \% \mathrm{CI})$ of TTNT comparing LDG versus SDG was 1.33 (1.21, 1.47), 1.20 (1.05, 1.39), 2.11 (1.80, 2.48), $1.12(0.98,1.27)$ for $\mathrm{Vd}, \mathrm{VRd}, \mathrm{CyBorD}$, and 


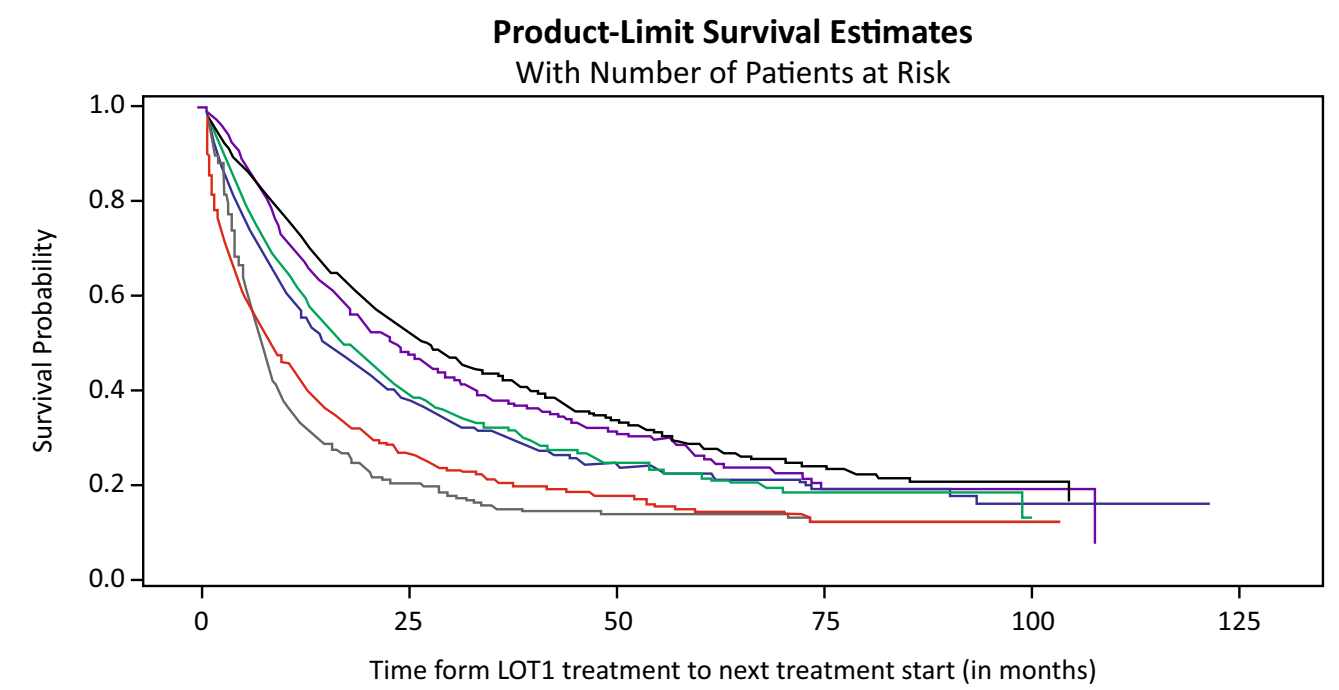

\begin{tabular}{|lll|}
\hline 1. CyBorD & 2. Other bortezomib containing & 3. Other \\
4. Rd & 5. VRd & 6. Vd \\
\hline
\end{tabular}

Patient at risk

$\begin{array}{cccc}1 & 1225 & 52 & 8 \\ 2 & 2481 & 146 & 38 \\ 3 & 2821 & 280 & 74 \\ 4 & 4358 & 735 & 197 \\ 5 & 2718 & 373 & 83 \\ 6 & 4128 & 485 & 107\end{array}$

Fig. 3 Time to next treatment (TTNT) in new patients at risk. $R d$ lenalidomide \pm dexamethasone, $V d$ bortezomib \pm dexamethasone, $V R d$ bortezomib + lenalidomide \pm dexamethasone, $C y B o r D$ cyclophosphamide + bortezomib + dexamethasone; bortezomib is present

other bortezomib-containing regimens, respectively. Rd was the only exception [0.68 (0.61, 0.76)] (Table 4).

\section{DISCUSSION}

The present population-based analysis in the United States evaluated the treatment patterns and OS in patients who had not received ASCT for MM. Treatment of this population is specifically challenging considering the presence of renal, hepatic, cardiac, and other comorbidities [15]. Identifying multiple myeloma patients who are not eligible for ASCT from a retrospective cohort study is not straightforward. SEER-Medicare and the Optum databases do not provide clinical detail information such as stage of MM, cytogenetic abnormalities, performance status such as Eastern Cooperative Oncology Group (ECOG) performance score, or frailty status to determine if patients are ASCT-ineligible. Although ASCT history is not equivalent to ASCT eligibility, this study provided useful information for ASCTineligible patients. Frontline therapies received by patients in the study were consistent with NCCN guideline recommendations for patients who did not receive ASCT for MM [6]. Bortezomib and lenalidomide-containing therapies 


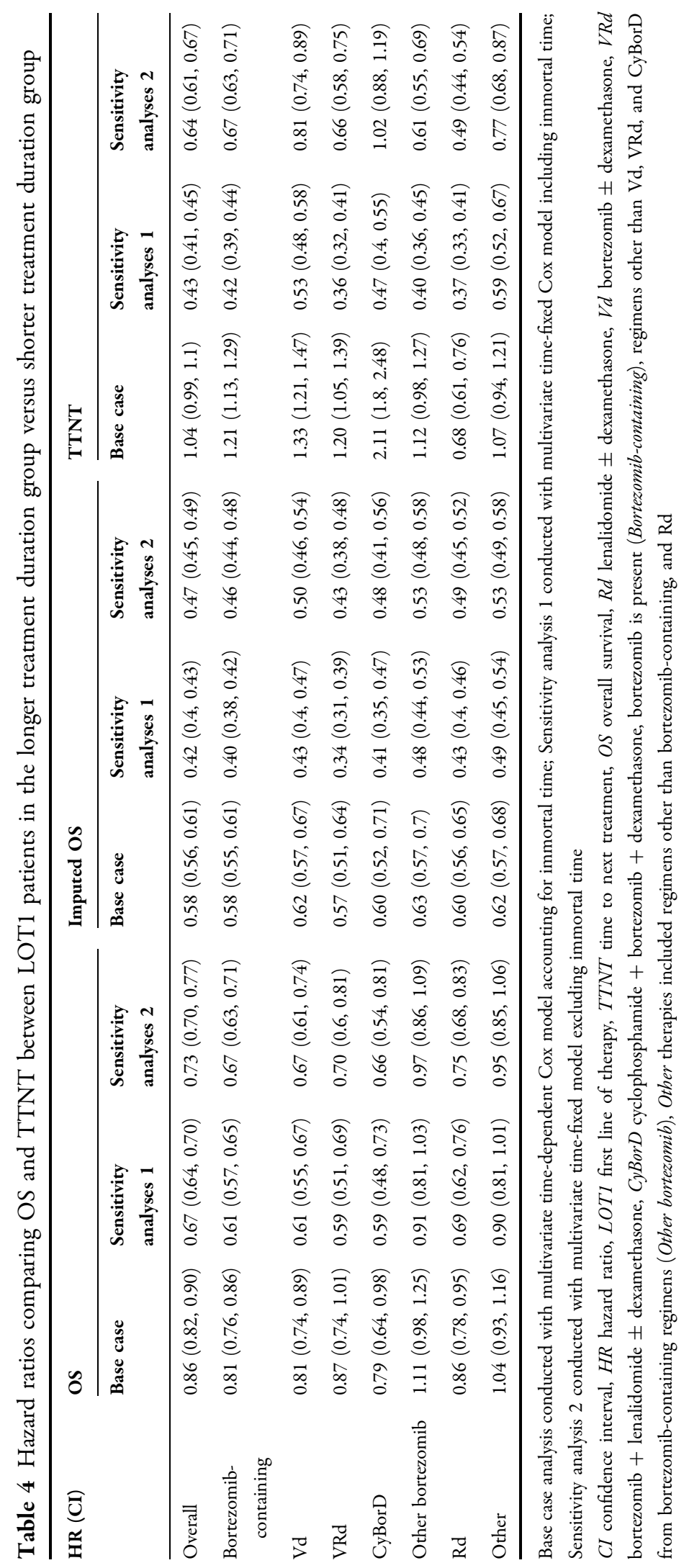


such as Rd, Vd, VRd and CyBorD were the most common treatment regimens in frontline. Still, subsequent therapies were mainly proteasome inhibitors and immunomodulatory-based treatment during the study period. Recently approved agents such as daratumumab, carfilzomib, and ixazomib were rare. Less than half of the patients received subsequent therapies, indicating substantial unmet need in this patient population.

Results of the propensity score matching and regression adjustment with age, gender, and comorbidities consistently demonstrated favorable OS among patients who received VRd therapy. While ascertaining the statistical significance associated with the treatment effectiveness of VRd, we have used Bonferroni correction to avoid any possibility of bias due to type-I error as a result of multiple testing. Hence, our results in favor of VRd over other competing regimens should be deemed conservative. A phase 3 study (SWOG s0777) evaluating the effect of VRd versus Rd in patients who did not receive ASCT for MM showed significantly higher median OS with VRd compared to Rd [75 months vs. 64 months, HR (95\% CI) 0.71 $(0.53,0.96)][16]$. Durie et al. reported a statistically significant improvement in OS [0.75 $(0.58,0.98)]$ during a 7 -year follow-up among patients with MM treated with VRd as compared with $\mathrm{Rd}$ therapy with age, intent to transplant, and ISS-adjusted [17]. Half of the patients included in this study were either frail or age $\geq 65$ years. The results of patients who did not receive ASCT were not reported. Our results of 0.83 comparing VRd versus $\mathrm{Rd}$ is comparatively relative worse compared to SWOG s0777. However, a multivariate regression model may not be sufficient when two different patient populations were mixed in one cohort without considering effect modification [17]. In addition, shorter duration of both VRd and $\mathrm{Rd}$ treatment in the real world, under-reporting of deaths in the Optum databases, and different subsequent therapy makes it difficult to directly compare with the SWOG s0777 study results. Except for SWOG S0777, there are no other head-to-head phase 3 studies comparing VRd with other frontline regimens in patients who are not eligible for ASCT. However, our result is consistent with a study [18] based on the US Flatiron Health EHR which reported the OS HR for VRd versus Rd as 0.88 (95\% CI 0.69, 1.11).

The longest TTNT was observed in patients treated with Rd therapy (24.3 months) compared with VRd therapy (20.4 months) and Vd therapy (15.4 months). Similar to the present study, Chari et al. reported that patients treated with Rd therapy (50.7 months) demonstrated a significantly longer TTNT compared with VRd therapy (35.7 months) and CyBorD therapy (22.3 months) [19]. The study was based on a claims database which is well known for the limitation of reporting death. However, the trends based on HRs between Chari et al. and the present study were similar. Often, Rd is used as maintenance therapy and administered to patients with comorbidities or high frailty [20]. It is possible that such patients would be reluctant to initiate another therapy which could potentially be more toxic, and hence decide to adhere to Rd, resulting in the long TTNT that was observed in the present study. More studies on TTNT of Rd in this setting are warranted. Although a longer treatment duration may not alleviate the patient's condition, it was associated with better survival outcomes [21]. Previous researchers have asserted the use of timedependent Cox model to be a common approach for adjusting immortal time bias $[12,22]$. The presence of immortal time was seen in the sensitivity analysis where the timefixed Cox model showed less favorable outcomes in the LDG upon exclusion of the immortal time from the LDG without it being added to the SDG. Compared to the time-fixed Cox models included in the sensitivity analyses, the base case analysis conducted with the timedependent Cox model correcting the misclassified immortal time inflated the differential risk of outcomes associated with LDG over SDG. Hence, our results are conservative, as consistency in treatment effect (i.e., inflation of HR) is observed in both the base case analysis and the sensitivity analysis addressing the immortal time bias. Studies have demonstrated that patients often discontinue frontline therapy due to disease progression, side effects or comorbidities, no evidence of disease, or due to 
financial constraints $[23,24]$. As the cause of treatment discontinuation was not captured in the database, it cannot be determined whether better outcomes were due to longer treatment, or with the fact that patients with fewer comorbidities and better overall health could better tolerate frontline treatment. Nevertheless, if there is no clinical justification, patients should be encouraged to comply on their treatment assignment.

Our results also indicated high comorbidity rates during frontline treatment. Comorbidities observed during the present study included anemia, peripheral neuropathy, nausea, fatigue, and diarrhea. The varying extent of toxicity and comorbidities experienced by patients between RCTs and real-world studies could also be related to the differing patient populations and characteristics; treatment center (academic vs. community) and associated number of experienced physicians; lack of protocol-directed treatment; and issues such as convenience, practicality, and direct and indirect costs [25]. Furthermore, these differences greatly impact the drug response, compliance to treatment, and generalizability of the results [26]. Several earlier studies have reported similar comorbidities with bortezomib treatment, which include neuropathic pain, sensory neuropathy, and fatigue [8, 27]. A recent meta-analysis reported that addition of bortezomib to the treatment regimens of patients with MM significantly improved outcomes; however; comorbidities associated with a longer duration of bortezomib therapy must be considered prior to treatment initiation [28].

There were several limitations of the present study. The partial reporting of deaths in the Optum databases and the long time lag for reporting in the SEER-Medicare database inflated the survival rate of patients receiving different therapies in these two databases. As $80 \%$ of the patients were from Optum, the partial data regarding death was a major limitation. Therefore, results of the present study must be interpreted with caution. However, sensitivity analyses were performed to estimate the imputed OS with consistent HRs compared to the base case. Residual confounding is possible after matching or regression adjustment, as important prognostic factors such as ECOG performance status, myeloma type, and cytogenetic risk are lacking in the databases used in this study. Subsequent therapy is a confounding factor for survival but not adjusted in the OS analysis. Bias away from the null may persist if VRd patients were followed by innovative agents such as daratumumab. Comparison of OS by frontline treatment represents the OS after receiving different treatment pathways led by the frontline treatment. The reason for treatment discontinuation is unknown. Patients who demonstrated a negative prognosis might not have tolerated bortezomib therapy for a longer duration of time. Treatment information was from claims databases including SEERMedicare, it is unclear whether patients were compliant to their oral treatment. In addition, data related to over-the-counter medications received by the patients were not available. Missing claims might have occurred if patients covered by a commercial insurance plan switched between plans. Changes to the treatment of patients with MM after the end of the study (SEER-Medicare: Dec 2016 and Optum: Sep 2018) have not been reported in the present study. Lack of confirmation regarding smoldering $\mathrm{MM}$, the doses of injectable, and detail patterns of maintenance therapy may be considered as a limitation of the study.

\section{CONCLUSION}

Results of the present study demonstrated that the frontline therapies prescribed to most patients who did not receive ASCT for MM in the United States were consistent with NCCN guideline recommendations. Those who received VRd showed better outcomes compared with Rd, Vd, and other bortezomib-based regimens, as measured by survival duration, although not statistically significant compared to CyBorD, after adjusting for age and comorbidities. The prevalence of morbidities in the 6-month lookback period prior to frontline treatment was substantial. Statistical models considering immortal time demonstrated that longer treatment is generally associated with longer OS compared with patients who received 
treatment for a shorter duration of time, particularly for first-line therapy as a whole.

\section{ACKNOWLEDGEMENTS}

Funding. This study and the journal's Rapid Service and Open Access fees were funded by Janssen Research \& Development, LLC.

Medical Writing Assistance. Writing and editorial assistance in the preparation of this article was provided by Gurpreet Virya and Rishabh Pandey, PhD of SIRO Clinpharm. Support for this assistance was provided by Janssen R\&D LLC.

Authorship. All authors meet the International Committee of Medical Journal Editors (ICMJE) criteria for authorship for this article, take responsibility for the integrity of the work as a whole, and have given their approval for this version to be published.

Disclosures. JH, RVR, LL, YL, PH, SN, SSS, CA, QL, MM, and UR are employees of Janssen R\&D, LLC, and may own or have options to own stock in the company. LS, LQ, AD, and RP report no conflict of interest. PZ was an employee of Janssen R\&D, LLC at the time of study and is currently employed at David Geffen School of Medicine, UCLA.

Compliance with Ethics Guidelines. The use of SEER registry and Optum (Clinformatics and EHR) databases was reviewed by the Institutional Review Board (IRB). The study protocol developed for obtaining data from the SEER Medicare database was reviewed and exempt by Advarra IRB (Columbia, MD), as this research project did not involve human patient research.

Data Availability. Data sharing is not applicable to this article as no datasets were generated or analyzed during the current study.

Open Access. This article is licensed under a Creative Commons Attribution-NonCommercial 4.0 International License, which permits any non-commercial use, sharing, adaptation, distribution and reproduction in any medium or format, as long as you give appropriate credit to the original author(s) and the source, provide a link to the Creative Commons licence, and indicate if changes were made. The images or other third party material in this article are included in the article's Creative Commons licence, unless indicated otherwise in a credit line to the material. If material is not included in the article's Creative Commons licence and your intended use is not permitted by statutory regulation or exceeds the permitted use, you will need to obtain permission directly from the copyright holder. To view a copy of this licence, visit http:// creativecommons.org/licenses/by-nc/4.0/.

\section{REFERENCES}

1. Bergsagel PL. Where we were, where we are, where we are going: progress in multiple myeloma. Am Soc Clin Oncol Educ Book. 2014;20:199-203.

2. Gerecke C, Fuhrmann S, Strifler S, Schmidt-Hieber M, Einsele H, Knop S. The diagnosis and treatment of multiple myeloma. Dtsch Arztebl Int. 2016;113: 470-6.

3. Cowan AJ, Allen C, Barac A, Basaleem H, Bensenor I, Curado MP, et al. Global burden of multiple myeloma: a systematic analysis for the global burden of disease study 2016. JAMA Oncol. 2018;4: 1221-7.

4. American Cancer Society. Key statistics about multiple myeloma 2019 [01 July 2019]. https://www. cancer.org/cancer/multiple-myeloma/about/keystatistics.html.

5. Fonseca R, Abouzaid S, Bonafede M, Cai Q, Parikh $\mathrm{K}$, Cosler L, et al. Trends in overall survival and costs of multiple myeloma, 2000-2014. Leukemia. 2017;31:1915-21.

6. Mikhael J, Ismaila N, Cheung MC, Costello C, Dhodapkar MV, Kumar S, et al. Treatment of multiple myeloma: ASCO and CCO joint clinical practice guideline. J Clin Oncol. 2019;37:1228-63.

7. Moreau P, Attal M, Facon T. Frontline therapy of multiple myeloma. Blood. 2015;125:3076-84. 
8. Richardson PG, Weller E, Lonial S, Jakubowiak AJ, Jagannath S, Raje NS, et al. Lenalidomide, bortezomib, and dexamethasone combination therapy in patients with newly diagnosed multiple myeloma. Blood. 2010;116:679-86.

9. Kumar SK, Callander NS, Hillengass J, Liedtke M, Baljevic M, Campagnaro E, et al. NCCN guidelines insights: multiple myeloma, version 1.2020. J Natl Compr Canc Netw. 2019;17:1154-65.

10. Kumar SK, Callander NS, Alsina M, Atanackovic D, Biermann JS, Castillo J, et al. NCCN guidelines insights: multiple myeloma, Version 3.2018. J Natl Compr Canc Netw. 2018;16:11-20.

11. Yang X, Kong AP, Luk AO, Ozaki R, Ko GT, Ma RC, et al. Validation of methods to control for immortal time bias in a pharmacoepidemiologic analysis of renin-angiotensin system inhibitors in type 2 diabetes. J Epidemiol. 2014;24:267-73.

12. Suissa S. Immortal time bias in observational studies of drug effects. Pharmacoepidemiol Drug Saf. 2007;16:241-9.

13. Li G, Taljaard M, Van den Heuvel ER, Levine MA, Cook DJ, Wells GA, et al. An introduction to multiplicity issues in clinical trials: the what, why, when and how. Int J Epidemiol. 2017;46:746-55.

14. Vickerstaff V, Omar RZ, Ambler G. Methods to adjust for multiple comparisons in the analysis and sample size calculation of randomised controlled trials with multiple primary outcomes. BMC Med Res Methodol. 2019;19:129.

15. Girnius S, Munshi NC. Challenges in multiple myeloma diagnosis and treatment. Leuk Suppl. 2013;2:S3-9.

16. Durie BG, Hoering A, Abidi MH, Rajkumar SV, Epstein J, Kahanic SP, et al. Bortezomib with lenalidomide and dexamethasone versus lenalidomide and dexamethasone alone in patients with newly diagnosed myeloma without intent for immediate autologous stem-cell transplant (SWOG S0777): a randomised, open-label, phase 3 trial. Lancet. 2017;389:519-27.

17. Durie BGM, Hoering A, Sexton R, Abidi MH, Epstein J, Rajkumar SV, et al. Longer term follow-up of the randomized phase III trial SWOG S0777: bortezomib, lenalidomide and dexamethasone vs. lenalidomide and dexamethasone in patients (Pts) with previously untreated multiple myeloma without an intent for immediate autologous stem cell transplant (ASCT). Blood Cancer J. 2020;10:53.
18. Durie BGM, Kumar SK, Usmani SZ, Nonyane BAS, Ammann EM, Lam A, Kobos R, Maiese EM, Facon T. Daratumumab-lenalidomide-dexamethasone vs. standard-of-care regimens: efficacy in transplantineligible untreated myeloma. Am J Hematol. 2020. https://doi.org/10.1002/ajh.25963.

19. Chari A, Parikh K, Ni Q, Abouzaid S. Treatment patterns and clinical and economic outcomes in patients with newly diagnosed multiple myeloma treated with lenalidomide- and/or bortezomibcontaining regimens without stem cell transplant in a real-world setting. Clin Lymphoma Myeloma Leuk. 2019;20:20.

20. Dempsey JL, Johns A, Rosko AE, Lazarus HM. The pharmacologic management of multiple myeloma in older adults. Expert Opin Pharmacother. 2019;20:887-902.

21. Rajkumar SV. Treatment of multiple myeloma. Nat Rev Clin Oncol. 2011;8:479-91.

22. Levesque LE, Hanley JA, Kezouh A, Suissa S. Problem of immortal time bias in cohort studies: example using statins for preventing progression of diabetes. BMJ. 2010;340:b5087.

23. Schwartz R, Davidson T. Pharmacology, pharmacokinetics, and practical applications of bortezomib. Oncology (Williston Park). 2004;18:14-21.

24. Sivaraj D, Green MM, Li Z, Sung AD, Sarantopoulos $S$, Kang Y, et al. Outcomes of maintenance therapy with bortezomib after autologous stem cell transplantation for patients with multiple myeloma. Biol Blood Marrow Transplant. 2017;23:262-8.

25. Richardson PG, San Miguel JF, Moreau P, Hajek R, Dimopoulos MA, Laubach JP, et al. Interpreting clinical trial data in multiple myeloma: translating findings to the real-world setting. Blood Cancer J. 2018;8:109.

26. Monti S, Grosso V, Todoerti M, Caporali R. Randomized controlled trials and real-world data: differences and similarities to untangle literature data. Rheumatology (Oxford). 2018;57:vii54-8.

27. Aguiar PM, de Mendonca LT, Colleoni GWB, Storpirtis S. Efficacy and safety of bortezomib, thalidomide, and lenalidomide in multiple myeloma: An overview of systematic reviews with meta-analyses. Crit Rev Oncol Hematol. 2017;113:195-212.

28. Sun CY, Li JY, Chu ZB, Zhang L, Chen L, Hu Y. Efficacy and safety of bortezomib maintenance in patients with newly diagnosed multiple myeloma: a meta-analysis. Biosci Rep. 2017;20:37. 